\title{
Modelo Conceitual do Objeto Virtual de Aprendizagem Colaborativa para Aprendizagem de Estatística (Collabora)
}

\author{
Eliana Cláudia Mayumi Ishikawa - UTFPR - eishikawa@utfpr.edu.br \\ Lucas Novelli - UTFPR - lucasnovelli@outlook.com \\ Vinícius Camargo Andrade - UTFPR - vcandrade@utfpr.edu.br \\ Simone Nasser Matos - UTFPR - snasser@utfpr.edu.br \\ Guataçara dos Santos Junior - UTFPR - guata@utfpr.edu.br
}

\begin{abstract}
RESUMO: Este trabalho apresenta o modelo conceitual de um objeto virtual de aprendizagem para o ensino da estatística no ensino superior (Collabora) contendo quatro módulos principais: Login, Colaboração, Aprendizagem e Chat e usa como base a aprendizagem colaborativa apoiada por computador. A diferença do objeto proposto em relação a outros trabalhos da literatura é que o mesmo oferece um controle automatizado para verificar se os alunos que pertencem a um grupo estão colaborando ao realizar uma atividade, composta por um conjunto de exercícios, proposta pelo professor. A construção e implementação do objeto é feita usando orientação a objetos o que fornece reusabilidade de análise, projeto e código. O Collabora permite ao professor realizar atividades que promova a verificação e integração entre os elementos de um grupo, avaliação da colaboração, avaliação da atividade e mapeamento da colaboração individual e em grupo.
\end{abstract}

Palavras-chaves: Objeto de Aprendizagem. Estatística. Colaboração. CSCL. Orientação a Objetos.

\begin{abstract}
This paper presents the conceptual model of a virtual learning object to the teaching of statistics in higher education (Collabora) containing four main modules: Login, Collaboration, Learning and Chat and uses based collaborative learning supported by computer. Unlike the proposed object in relation to other published work is that it provides an automated control to verify that students who belong to a group are collaborating to perform an activity consisting of a set of exercises proposed by the teacher. The construction and implementation of the object is made using object orientation which provides analysis of reusability, design and code. The Collabora allows the teacher to carry out activities to promote the verification and integration between the elements of a group, evaluation of collaboration, evaluation of activity and mapping of individual collaboration and group.
\end{abstract}

Keywords: Learning Object. Statistic. Collaboration. CSCL. Object orientation.

\section{Introdução}

Estudos indicam que a aprendizagem dos alunos melhora quando se utiliza de forma agregada, a teoria, os experimentos, realidade do aluno e as tecnologias computacionais (BARROQUEIRO; AMARAL; DE OLIVEIRA, 2013), assim, as tecnologias de informação e comunicação (TIC) podem ser usadas como ferramenta de apoio ao ensino e aprendizagem de Estatística, uma vez que favorecem o processo de aprendizagem, especialmente se contém um processo comunicacional interativo, dinâmico e bilateral entre o professor e alunos e entre os próprios alunos.

O uso integrado de tecnologias computacionais, tais como os objetos de aprendizagem (OA) e a Aprendizagem Colaborativa Apoiada por Computadores (CSCL) é uma alternativa capaz de proporcionar melhorias significativas no processo de aprendizagem, oferecendo mais e melhores condições ao aluno para participar ativamente do processo de construção de seu próprio conhecimento e do grupo como um todo, conforme relatado nas pesquisas desenvolvidas por Fujii e Silveira (2006), Cunha (2009), Oliveira e Tedesco (2010), Cardoso (2010), entre outros.

Este trabalho descreve o modelo conceitual para o objeto virtual de aprendizagem colaborativo (Collabora) que possibilita o reuso de código, análise e projeto. A implementação do modelo cria um objeto capaz de medir a colaboração entre pares em domínios diferentes, bem como permite maior agilidade no processo de obtenção de dados quantitativos sobre a aprendizagem referente ao indivíduo ou ao grupo.

O Collabora permite ao professor criar atividade compostas por um conjunto de exercícios que são classificados de acordo com a ementa, conteúdo e grau de 
complexidade. Ao final da atividade, é informado um score de acordo com a atividade proposta e poderá ser feito o mapeamento da colaboração alcançada de forma individual e pelo grupo, assim como um levantamento da percepção colaborativa do grupo em relação aos demais integrantes.

\section{TIC e a Educação Estatística}

Apesar dos avanços da aplicação e no desenvolvimento das Tecnologias de Informação e Comunicação (TIC), há necessidade de transformações nas práticas desenvolvidas na sala de aula, na apropriação das TIC e na visão que os alunos apresentam sobre os conceitos a serem trabalhados (SILVA; SCHIMIGUEL, 2014).

Para Kenski (2007), é preciso incorporar a tecnologia à sala de aula, aos métodos de ensino e aprendizagem e ao cotidiano dos alunos, uma vez que os vínculos entre o conhecimento, poder e tecnologias estão presentes em todas as épocas e em todos os tipos de relações sociais.

No âmbito da Educação Estatística, as TIC auxiliam o processo de ensino e aprendizagem de forma sistemática e quando esses forem utilizados de forma estratégica no processo de ensino e aprendizagem, podem auxiliar os educadores e discentes a compartilharem problemas, perspectivas, ideias e soluções (BARROQUEIRO; AMARAL; OLIVEIRA, 2011).

Assim, tais tecnologias podem se caracterizar como uma ferramenta de apoio ao ensino de Estatística, pois segundo Burril apud Estevam e Kalinke (2013, p. 106), "torna acessível a todos os alunos a Estatística e o raciocínio estatístico, fornece pistas sobre como trabalhar com dados e torna possível a utilização de dados reais e situações de interesse que motivam estudos estatísticos e matemáticos”.

Apesar dos dados positivos assinalados pelos diversos autores em relação ao ensino e na aprendizagem de Estatística pautado em TIC, constatou-se que as pesquisas não apontaram a criação de novas ferramentas que possam alavancar a relação ensino e aprendizagem fora do tradicional, uma vez que se fizeram uso tecnologias já existentes no mercado.

Esse é um aspecto importante quando se tem como objetivo utilizar as TIC como ferramentas ou recursos que permitem novas formas de acesso ao conhecimento e de relacionamento entre conteúdos estatísticos e atores do processo educacional. Haja vista que o desenvolvimento de novas tecnologias pode ser uma das maneiras de se que se busca a inovação na concepção do processo educativo, do desenvolvimento de novas estratégias de ensino e de aprendizagem, de práticas mais flexíveis, em termos de: tempo, espaço, conteúdos e processos (BARROS et al.,2011).

É neste contexto que surgem os Objetos de Aprendizagem (OA), que permitem desenvolver ferramentas e ambientes de aprendizagem suportados pelas TIC.

\section{Aprendizagem Colaborativa Apoiada por Computador - Computer Supported Collaborative Learning (CSCL)}

A Aprendizagem Colaborativa Apoiada por Computadores (CSCL) tem como premissa viabilizar ambientes computacionais que disponibilizem mecanismos que apoiem diversas etapas do processo social de construção de conhecimento. É a ciência que estuda como as pessoas podem aprender em grupo com o auxílio do computador, podendo ser aplicada em todos os níveis da educação formal, desde o jardim de infância até a graduação (STAHL et al., 2006).

A CSCL pode ser tratada como uma estratégia de aprendizado na qual pequenos grupos, cada um com diferentes níveis de habilidades e conhecimento, é responsável pela sua própria aprendizagem, assim como pela aprendizagem de todo o grupo, por meio de 
3

uma rede de interações sociais entre os envolvidos para a construção de um objetivo comum na qual a colaboração é ativa e a avaliação de todos é essencial.

A CSCL utiliza as mesmas estratégias educativas e dispõe de recursos computacionais como ferramentas mediadores do processo de ensino-aprendizagem do grupo. $\mathrm{O}$ suporte dado pelo computador à aprendizagem colaborativa, por meio dessas ferramentas, visa melhorar a dinâmica entre os membros do grupo, possibilitando aos mesmos alcançarem objetivos mais ricos em conteúdo, na medida em que reúne propostas e soluções de vários alunos do grupo, o que incentiva o compartilhamento de informações e a responsabilidade sobre sua veracidade.

$\mathrm{O}$ uso dos recursos computacionais como uma ferramenta educacional traz vantagens quando esse é feito por grupos de alunos, uma vez que possibilita a troca de ideias entre si e entre os grupos, propiciando a construção do aprendizado coletivo e em equipe, sendo que as habilidades desenvolvidas ocorrem naturalmente, sempre sob o gerenciamento do professor (GIANOTTO; DINIZ, 2010).

\section{Objeto de Aprendizagem}

Wiley (2000), descreve os OA como elementos de um novo tipo de instrução por meio do computador, que tem como premissa o paradigma de orientação a objetos, utilizado na área de ciência da computação. No paradigma de orientação a objetos, os objetos são representações de abstrações de entidades do mundo real e podem ser implementadas com o uso de tecnologias de construção de software por meio do reuso de componentes (SOUZA et al.,2007), assim percebe-se que uma das consequências desse tipo de abordagem é a melhoria da produtividade no processo de trabalho, uma vez que, a cada novo projeto, não é necessário recomeçar desde o seu início.

A premissa que envolve a criação de um OA é a de que esses sejam blocos que serão construídos ou alterados para serem usados e reutilizados em diversas áreas, disciplinas e conteúdos. Assim, os OA geralmente são criados em módulos que podem ser reusados em diferentes contextos de aprendizagem (curso online, aulas a distância, etc.).

Tais objetos são pequenas unidades de recursos de aprendizagem digitais construídos por meio de uma abordagem mais ampla, onde a construção pode ser realizada em qualquer mídia ou formato, ou ainda, limitar-se da combinação de HTML, Java e outras linguagens de programação. Assim, um OA pode ser desde uma simples animação até uma simulação computacional (FLÔRES, 2011; TAROUCO, 2003). São exemplos de objetos de aprendizagem: conteúdo multimídia, conteúdos instrucionais, objetivos de ensino e software instrucional (WILEY, 2000).

Considerando as diferentes definições para os objetos de aprendizagem, pode-se concluir que todas são resultados de diversas visões quanto a sua aplicação pedagógica e tecnológica, porém, algumas características comuns são esperadas para os OA (TAROUCO, 2006; GAZZONI et al., 2006): reusabilidade; adaptabilidade; modularidade; acessibilidade; durabilidade; interoperabilidade.

E consenso também entre os autores, que os objetos de aprendizagem (OA) se configuraram como uma ferramenta complementar na construção e fixação de conceitos desenvolvidos junto aos alunos. Assim, esses recursos podem ser utilizados para facilitar o processo de aprendizagem, pois tendem a proporcionar a obtenção de conhecimento de maneira mais motivadora o que torna as aulas mais atrativas, com vista a maximizar as situações de aprendizagem onde o recurso possa ser utilizado.

A criação de um objeto de aprendizagem é uma tarefa que exige um trabalho colaborativo importante, pois permite sua reutilização em vários contextos de aprendizagem e é sempre estruturado por um conteúdo, seja por uma unidade curricular de um curso, uma aula ou até mesmo por uma atividade (GAZZONI et al., 2006). 


\section{Modelo do Objeto Virtual de Aprendizagem Colaborativa (Collabora) para Estatística}

Tendo como propósito melhorar o ensino de estatística e outras disciplinas por meio da colaboração dos alunos o Objeto Virtual de Aprendizagem Colaborativa (Collabora) para Estatística disponibilizará um meio rápido e prático para a realização das atividades colaborativas, por meio de chat, links e disponibilização de arquivos. O modelo proposto foi constituído por quatro módulos individuais: Login, Colaboração, Aprendizagem e Chat, descritos nas próximas seções.

\subsection{Módulo Login}

É responsável por manter os dados (incluir, consultar, alterar e excluir) dos usuários, gerenciar as permissões de acesso e importar dados de um arquivo texto contendo informações sobre $\mathrm{o}$ aluno, professor e administrador e essas são registradas automaticamente em uma base de dados. Todo acesso é realizado a partir de um login e senha, os quais são validados e as funcionalidades para a cada tipo de usuário (aluno, professor e administrador) é liberada. $\mathrm{O}$ aluno é definido por um nome, registro acadêmico, turma e senha. O professor possui um nome, senha, turmas e suas respectivas disciplinas. $\mathrm{O}$ administrador é formado apenas por um nome e senha. Independentemente do tipo de usuário que tenha realizado o login ou logoff, a informação é armazenada para identificar quais usuários estão ativos.

A Figura 1 ilustra o modelo conceitual do módulo de Login efetuado tanto por um aluno quanto por um professor. Ambos são identificados na figura pelo objeto [Usuário].

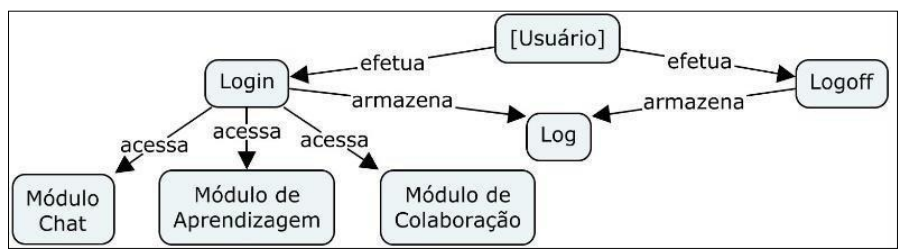

Figura 1 - Módulo Conceitual do Módulo Login

Ao efetuar login, o aluno e o professor possuem acesso aos módulos Chat, Aprendizagem e Colaboração. Será armazenado o log para que seja possível verificar quais usuários estão conectados. A administrador tem acesso aos módulos Login e Chat. A Figura 2 exibe o modelo conceitual após a realização do login pelo [Administrador].

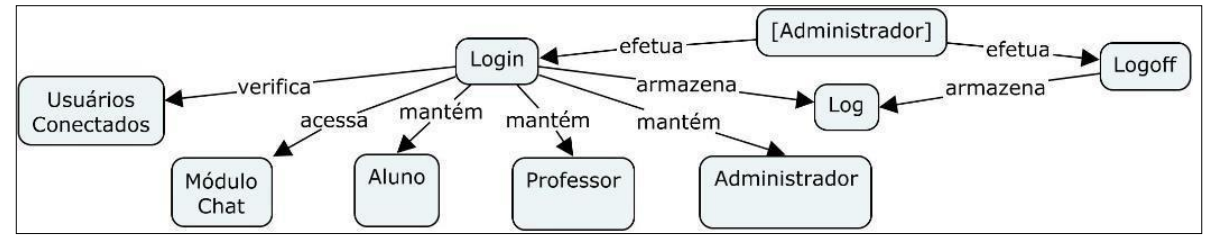

Figura 2 - Modelo Conceitual do Login de um Administrador

O [Administrador] possui permissão de verificar usuários conectados, manter cadastros de professores e manter outros administradores.

\subsection{Módulo Colaboração}

É responsável por implementar os conceitos de aprendizagem colaborativa, tais como: a) negociação entre os participantes do grupo, b) avaliação da colaboração durante o desenvolvimento de um exercício e após a finalização da atividade, c) gerenciamento estatístico, d) avaliação da colaboração pelos membros do grupo e e) cálculo da nota de colaboração por aluno e grupo. 


\section{Modelo do Objeto Virtual de Aprendizagem Colaborativa (Collabora) para} estabelecida quando existe divergência na resposta final. Neste caso, é realizado a votação para decidir qual a resposta que será adotada pelo grupo.

A avaliação da colaboração ocorre durante a finalização de uma atividade e após a execução de todas utilizando medidas presentes na literatura. O professor pode estabelecer um peso para cada medida de acordo com sua relevância, sendo que o valor total para a soma dos pesos deve ser 9 (nove).

Para mensurar e avaliar a colaboração, serão utilizadas medidas encontradas na literatura, onde alguns índices de colaboração e participação propostas por Ferraz et al. (2015), e outros critérios apresentados por Pietruchinski e Pimentel (2015), Khandaker; Soh (2010) e Dascalu et al. (2010) foram adaptadas para atender os propósitos desse trabalho.

Algumas medidas usadas são (DASCALU et al. (2010); FERRAZ et al. (2015)): durante e após a execução são: quantidade de mensagens postadas pelo grupo; Quantidade de mensagens significativas postadas por grupo; Quantidade de palavras escritas utilizadas na mensagem postadas pelo grupo, entre outras.

A avaliação da colaboração durante a execução de um exercício é necessária para que se possa verificar a colaboração dos alunos de forma individual em um determinado exercício. Essa ação é importante, pois um aluno pode colaborar de forma diferente em exercícios de uma mesma atividade, o que pode por exemplo, indicar ao professor as dificuldades do aluno em determinado conteúdo. Já a avaliação após a execução de uma atividade irá somar o número total da colaboração dos membros de um grupo em uma atividade. Esse valor será usado como referência para o calcular o escore da colaboração de cada integrante do grupo.

O gerenciamento estatístico é realizado com o objetivo de fornecer dados quantitativos sobre o processo de colaboração entre os alunos durante o desenvolvimento da atividade. Algumas medidas estatísticas para o Collabora são: Tempo gasto pelo grupo para realizar a atividade; Tempo gasto pelo grupo para realizar o exercício; Quantidade de acessos ao sistema por aluno; Total de links e arquivos compartilhados por aluno e grupo na atividade; Histórico e média da colaboração do aluno; entre outras.

Após o encerramento (entrega) de cada uma das atividades, os membros do grupo irão responder um questionário online (implementado no Collabora), composto por três questões que dizem respeito a sua auto-avaliação, avaliação de seus pares e do processo colaborativo.

Esta conterá os seguintes questionamentos usando como respostas a escala de Likert (1932) (0-2 muito pouco, 2-4 pouco, 4-6 média, 6-8 acima da média ou 9-10 muito): a) Qual foi o seu grau de colaboração durante a realização da atividade? b) Como você avalia a colaboração de cada integrante do seu grupo? c) Quanto a interação com os membros do seu grupo ajudou na realização da atividade?

O cálculo da nota de colaboração do aluno é dada considerando o resultado obtido pelo cálculo das medidas após a finalização da atividade (Peso 9 - distribuídos pelas medidas consideradas) somada com a avaliação que o aluno teve em relação aos seus pares (Peso 1) e dividida pela soma dos pesos. Por exemplo, se durante a realização da atividade teve-se:

- Medida: Quantidade de mensagens postadas por grupo considerando a atividade (Valor de referência)

- 100 mensagens postadas

- Medida: Quantidade de mensagens postadas por aluno considerando a atividade (Peso 1) 
6

- Se o aluno teve como quantidade de mensagem postadas $=10$ então ele colaborou $10 \%$. Neste caso, se ele tivesse postado 100 sua nota neste item seria 1 , porém como postou somente 10 , sua nota será 0,1 .

- Medida: Quantidade de palavras significativas utilizadas na mensagem postadas pelo grupo (Valor de referência)

○ 20 mensagens postadas significativa

- Medida: Quantidade de palavras significativas utilizadas na mensagem postadas pelo aluno (Peso 8)

- Se o aluno teve como quantidade de mensagem significativas postadas $=2$ então colaborou $10 \%$. Neste item sua nota será 0,8 .

- Se a nota do aluno pelos seus pares foi 5 , sua nota neste item será 0,5 .

- Portanto, a nota final de colaboração do aluno será $(0,1+0,8+0,5) / 10=0,14$.

Após o cálculo da nota de colaboração o professor irá utilizar o Quadro 1 para verificar o grau de colaboração do aluno na atividade.

Quadro 1 - Faixa de Valores para determinar o grau de colaboração

\begin{tabular}{|c|c|}
\hline Faixa de Valores & Grau de colaboração \\
\hline $0-2$ & Muito baixa colaboração \\
\hline $2-4$ & Baixa colaboração \\
\hline $4-6$ & Média colaboração \\
\hline $6-8$ & Boa colaboração \\
\hline $8-10$ & Muito boa colaboração \\
\hline
\end{tabular}

Considerando o exemplo anterior, o aluno analisado teve um grau de colaboração muito baixo na realização da atividade. Além disso, é de responsabilidade do módulo de colaboração verificar a interação dos participantes do grupo, bem como avisá-los quando não houver colaboração por parte dos mesmos. A Figura 3 mostra o modelo conceitual do módulo de colaboração.

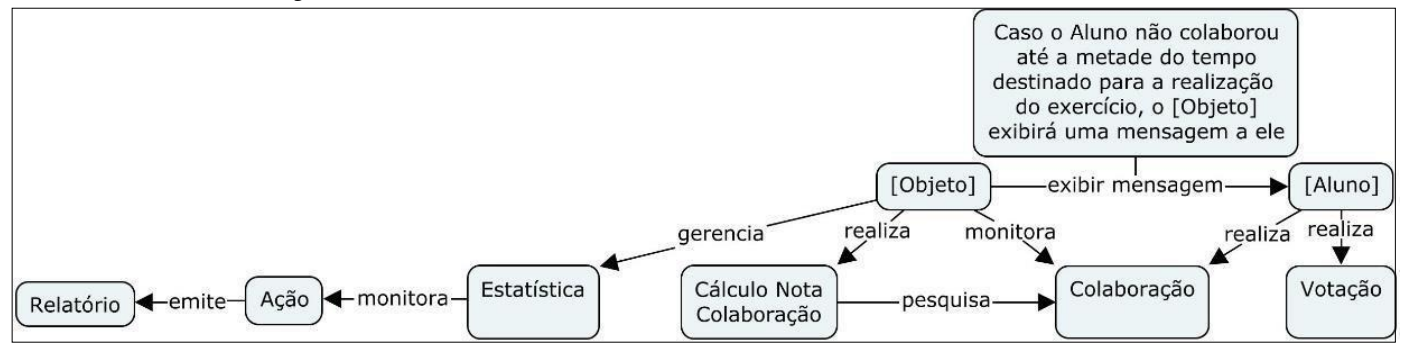

Figura 3 - Modelo conceitual do módulo de colaboração

\subsection{Módulo Aprendizagem}

Módulo responsável pela implementação dos requisitos relacionados a atividade, sendo estes: a) manter ementa, conteúdo, disciplina, exercício, turma e atividade; b) montar atividade; c) visualizar questões e atividades; d) clonar exercício; e) enviar resposta; f) correção resposta; g) contabilidade das respostas; h) feedback de questões e de atividade. As informações necessárias para manter os cadastros são:

- Disciplina: Composta por nome e um conjunto de ementas.

- Ementa: Possui nome e um conjunto de conteúdo.

- Conteúdo: Possui nome e uma descrição dos conteúdos abordados.

- Turma: Contêm um código, disciplina e um conjunto de alunos.

- Exercício: É composto obrigatoriamente por um enunciado, ementa, conteúdo, peso, dificuldade, um conjunto de no máximo sete e mínimo três proposições, uma referência e a dificuldade (baixa, média ou alta). Podem ser incluídos uma imagem para o enunciado e para cada proposição e um tempo fixo para a sua resolução. 
- Proposição: Contêm obrigatoriamente a definição da proposição, indexador que será identificado pelos números 01, 02, 04, 08, 16, 32 ou 64 e se a mesma é verdadeira ou falsa. Pode conter também uma imagem associada a definição da proposição.

- Atividade: Possui um nome, ementa, conjunto de questões, quantidade total de questões, quantidade de questões em função de sua dificuldade, data de início e fim e um conjunto de grupos.

- Grupo: Possui um nome e um conjunto de alunos com no mínimo dois e máximo quatro.

A Figura 4 ilustra o modelo conceitual do módulo de aprendizagem do ponto de vista do [Administrador], que pode manter Disciplina, Ementa e Turma. Estas podem ser cadastradas manualmente ou automaticamente. O cadastro manual indica que a inserção, exclusão e atualização, tanto de disciplina, quanto de ementa e turma, serão realizadas pelo próprio Administrador. Caso contrário, se a opção for pela maneira automática, o sistema fará a leitura de um arquivo que realizará as operações de inclusão, exclusão e atualização.

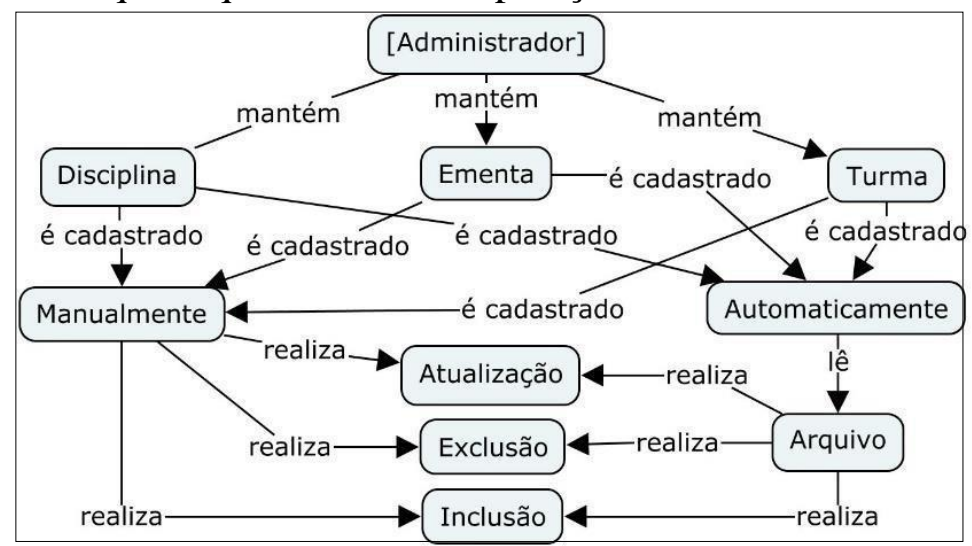

Figura 4 - Modelo Conceitual do Módulo de Aprendizagem - Visão Administrador

Exercícios, atividades e conteúdos são mantidos pelo [Professor] e devem obrigatoriamente cadastrados manualmente a fim de realizar sua inclusão, atualização e exclusão. Além disso, é de responsabilidade do professor realizar a clonagem de um exercício, caso seja necessário. O modelo conceitual das responsabilidades do [Professor] é ilustrado na Figura 5.

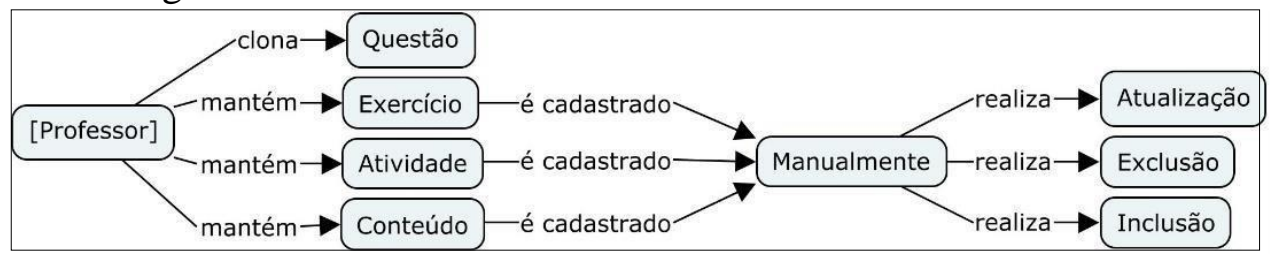

Figura 5 - Modelo Conceitual para Clonar um Exercício

$\mathrm{O}$ aluno no módulo de aprendizagem interage com o grupo para realizar as atividades, podendo visualizar as informações referente à atividade que estão sendo realizadas, tais como: questões faltantes, questões corretas e incorretas até o momento; visualizar exercício e enviar reposta.

A correção da resposta compara a resposta correta armazenada e a assinalada pelo aluno, se forem iguais, será computado um ponto, caso contrário, a pontuação será calculada utilizando a expressão (1):

$$
\mathrm{P}=\frac{\mathrm{NP}-(\mathrm{NTPC}-(\mathrm{NPC}-\mathrm{NPI}))}{\mathrm{NP}}
$$

Onde: $\mathrm{P}=$ pontuação do grupo no exercício; $\mathrm{NP}=$ número de proposições do exercício; $\mathrm{NTPC}=$ número total de proposições corretas; $\mathrm{NPC}=$ número de proposições corretas 


\section{8}

consideradas pelo grupo; NPI = número de proposições incorretas consideradas pelo grupo.

Após esta etapa, o sistema contabiliza e guarda os pontos de todas as questões durante a atividade, oferecendo o feedback ao grupo toda vez que um exercício é respondido, informando a resposta correta e a pontuação dos mesmos no exercício.

Ao fim de cada atividade, há também o fornecimento do resultado desta ao grupo. Neste momento, é gerado um relatório com todas as perguntas e suas respectivas respostas juntamente com a pontuação do grupo na atividade realizada, sendo possível ser baixado pelos alunos. Cada atividade possui uma nota de 0 a 10 , esta é composta pelos pontos obtidos da resolução dos exercícios. O modelo conceitual referente ao aluno no módulo de aprendizagem está ilustrado na Figura 6.

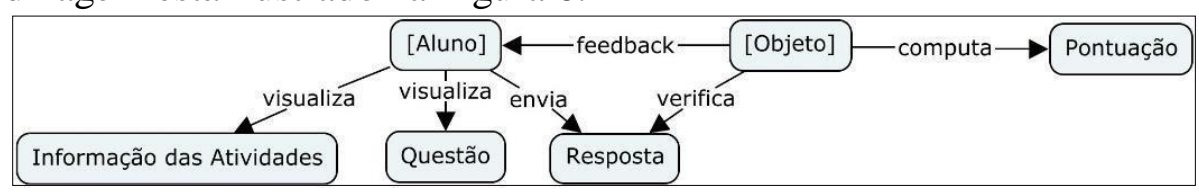

Figura 6 - Modelo conceitual do Módulo de Aprendizagem - Visão do Aluno

\subsection{Módulo Chat}

O Módulo de Chat é responsável por gerenciar as interações entre os integrantes do grupo e realizar a interface entre os usuários e os demais módulos. Seus principais requisitos são: envio e recebimento de mensagens, imagens e arquivos e liberação das atividades. A liberação das atividades ocorre apenas quando no mínimo 2 (dois) integrantes do grupo estiverem disponíveis no sistema, caso contrário a atividade não é liberada e o sistema deve informar aos demais integrantes quem não está disponível.

Este módulo possui limitações, como por exemplo, a troca de mensagens é permitida apenas entre participantes do mesmo grupo, não sendo permitido que dois grupos distintos interajam. Cada mensagem deve possuir o nome do aluno que a enviou e todos do grupo devem receber a mesma informação. Além disso, um integrante do grupo pode enviar uma mensagem ao professor, neste caso, os demais integrantes devem receber uma cópia da mensagem, e esta, deve ser sinalizada como enviada ao professor.

O professor possui acesso ao chat, porém suas funcionalidades são limitadas ao envio e recebimento de mensagens. Para uma melhor organização destas, as mesmas são agrupadas em diferentes conversas, considerando o grupo que elas pertençam. A Figura 7 ilustra como o módulo Chat funciona.

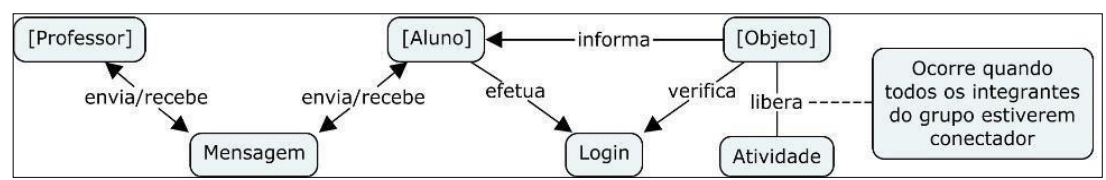

Figura 7 - Modelo Conceitual do Chat

\section{Considerais finais}

$\mathrm{O}$ modelo do objeto proposto (Collabora) permite ao aluno construir, interagir e colaborar com os seus pares durante a solução de atividade. É formado por 4 módulos: Login, Colaboração, Aprendizagem e Chat e está fundamentado na teoria CSCL que traz ganhos para o ensino e aprendizagem, neste trabalho na área de Estatística, uma vez que os alunos terão à disposição um objeto que permite: o contato mais próximo com a disciplina fora da sala de aula, contato com o professor, resolver problemas em grupo de forma colaborativa, feedback em tempo real sobre sua aprendizagem e opinião do grupo.

Em relação aos professores o modelo melhora a gestão de atividades, além de poder determinar o grau de colaboração que os alunos atingiram durante a realização da atividade, bem como estabelecer um score de aprendizagem por aluno e grupo. 
O Collabora está em fase de desenvolvimento e algumas tecnologias que estão sendo usadas para sua criação: Framework Struts 2.0 (APACHE SOFTWARE FUNDATION, 2016), responsável pela parte lógica de todo o sistema; Bootstrap (BOOSTRAP, 2016), responsável por padronizar as interfaces que interagirão com o usuário; PostgreSQL (POSTGRESQL, 2016), sistema gerenciador de banco de dados da aplicação; e Eclipse IDE (ECLIPSE, 2016), plataforma utilizada para o desenvolvimento da ferramenta. Sua modelagem de software está sendo feita usando a orientação a objetos e por isto permitirá maior reusabilidade de código, análise e projeto.

Com a implementação do Collabora, o mesmo se tornará um produto educacional para promover uma participação mais significativa dos alunos, tornando-os assim, participantes ativos do processo de aprendizagem para aquisição do conhecimento estatístico e probabilístico.

O diferencial do Collabora é que o mesmo não visa apenas criar um Objeto de Aprendizagem (OA) ou promover a colaboração, mas também medir a colaboração entre pares em domínios diferentes, bem como permitir maior agilidade no processo de obtenção de dados sobre a aprendizagem referente ao indivíduo ou ao grupo.

Os dados obtidos com uso do Collabora serão cruzados com os resultados das avaliações, observações e percepções adquiridas pelos pesquisadores durante as aulas de Probabilidade e Estatística. A análise e discussão desses resultados podem ser vistos como uma contribuição para a aprendizagem de Probabilidade e Estatística, já que a avaliação do produto educacional proposto será validada por seus pares, sob um enfoque pedagógico e computacional.

\section{Referências Bibliográficas}

APACHE SOFTWARE FUNDATION. Apache Struts. Disponível em: <https://struts.apache.org/>. Acesso em: 18 mai. 2016.

BARROQUEIRO, C.M; AMARAL, L. H; OLIVEIRA, C. A. S. O Uso Das Tecnologias da Informação e da Comunicação no Ensino de Ciências e Matemática. Revista Tecnologia \& Cultura - Rio de Janeiro - ano 19 - $\mathrm{n}^{\mathrm{o}} 13$ - pp. 45/58 - jul./dez. 2011.

BARROS, D.M.V.; et al. Educação e tecnologias: reflexão, inovação e práticas. Lisboa: [s/n], ISBN: 978-989-20-2329-8, 2011.

BOOSTRAP. Boostrap. Disponível em: <http://getbootstrap.com/>. Acesso em: 18 mai. 2016.

CARDOSO, A. L. M. de S. Construção e difusão colaborativa do conhecimento: uma experiência construtivista de educação em um ambiente virtual de aprendizagem. 2010. 305 f. Tese (Doutorado em Educação) - Universidade Federal da Bahia, Salvador (BA). Disponível em <https://repositorio.ufba.br/ri/bitstream/ri/11123/1/Tese\%20-\%20Antonio\%20Cardoso.pdf >. Acesso em: 20 out. 2015.

CUNHA, A. A. S. da. Definição de um modelo de sistema de aprendizagem colaborativa apoiado por computador com ênfase em trabalho em equipe. 2009. 146f. Dissertação (Mestrado em Engenharia Elétrica e de Computação) - Universidade Estadual de Campinas, Campinas (SP). Disponível em: <http://csbc2009.inf.ufrgs.br/anais/pdf/wie/st02_02.pdf. Acesso em: 25 ago. 2015.

DASCALU, M., TRAUSAN-MATU, S., and DESSUS, P. Utterances assessment in chat conversations. Research in Computing Science, 46:323-334, 2010.

ECLIPSE. Eclipse. Disponível em: <https://www.eclipse.org/downloads/>. Acesso em: 18 mai. 2016.

ESTEVAM, E.J. G;KALINKE, M.A. Recursos Tecnológicos e Ensino de Estatística na Educação Básica: um cenário de pesquisas brasileiras. Revista Brasileira de Informática na Educação, Volume 21, Número 2, 2013. 
FERRAZ, P O.; DE OLIVEIRA, P.; HORNINK, G.G. Desenvolvimento e Implementação de Indicadores de Colaboração e Participação no Moodle. Informática na educação: teoria \& prática, v. 18, n. 1, P. $85-95,2015$.

FLÔRES, M. L. P. Metodologia para criar objetos de aprendizagem em matemática usando a combinação de ferramentas de autoria. 2011. 140 f. Tese (Doutorado em Informática na Educação), Centro de Estudos Interdisciplinares em Novas Tecnologias na Educação, Universidade Federal do Rio Grande do Sul, 2011. Disponível em: < http://www.lume.ufrgs.br/handle/10183/39669>. Acesso em: 4 jun. 2015.

GAZZONI, A. et. al. Proporcionalidade e Semelhança: aprendizagem via objetos de aprendizagem. RENOTE: Revista Novas Tecnologias na Educação. Porto Alegre, v. 4 n. 2, p. 1-09, 2006. Disponível em http://seer.ufrgs.br/renote/article/view/14141/8076. Acesso em 11 de out. 2015.

GIANOTTO, D. E. P.; DINIZ, R. E. DA S. Formação inicial de professores de Biologia: a metodologia colaborativa mediada pelo computador e a aprendizagem para a docência. Ciência \& Educação, Bauru, v. 16, n. 3, p. 631-648, 2010. Disponível em: <http://www.scielo.br/pdf/ciedu/v16n3/v16n3a09>. Acesso em: 25 mai. 2015.

KENSKI, Vani Moreira. Educação e tecnologias: o novo ritmo da educação. São Paulo: PAPIRUS2010, 2007.

KHANDAKER, N. and SOH, L.-K. Classroomwiki: A collaborative wiki for instructional use with multiagent group formation. Learning Technologies, IEEE Transactions on, 3(3):190-202, 2010.

LIKERT, Rensis. A Technique for the Measurement of Attitudes. Archives of Psychology, n.140, p. 1-55, 1932.

OLIVEIRA, E. A.; TEDESCO, P. i-collaboration: Um modelo de colaboração inteligente personalizada para ambientes de EAD. Revista Brasileira de Informática na Educação, v. 18, n. 1, p. 17-31, 2010. Disponível em: <http://www.cesar.org.br/site/files/uploads/2012/10/1213-1510-3PB.pdf $>$. Acesso em: 12 abr. 2015.

PIETRUCHINSKI, M. H. E PIMENTEL, A. R. (2015). An architectural model of multi-agent systems for student evaluation in collaborative game software. In : ICAISC 2015: International Conference on Articial Intelligence and Soft Computing, Berlin, Germany, (Sep 14-15, 2015), volume 2, paginas 1542. World Academy of Science, Engineeringand Technology, World Academy of Science, Engineering and Technology.

POSTGRESQL. PostgreSQL. Disponível em <http://www.postgresql.org/>. Acesso em: 18 mai. 2016.

SILVA, J. F.; SCHIMIGUEL, J. Proposta para o uso das TICs na construção de um ambiente de aprendizagem norteado pelos conceitos da educação estatística no ensino superior. Revista de Produção Discente em Educação Matemática. ISSN 2238-8044, v. 3, n. 1, 2014.

SOUZA, A. R. de; YONEZAWA,W. M.; SILVA, P. M.. Desenvolvimento de Habilidades em Tecnologia da Informação e Comunicação (TIC) por meio de Objetos de Aprendizagem. In: PRATA, Carmem Lúcia et al. Objetos de aprendizagem: uma proposta de recurso pedagógico/Organização. Brasília: e-book, p.49-59, 2007.

STAHL, G., KOSCHMANN, T.; SUTHERS, D. (2006). Computer-supported collaborative learning: An historical perspective. In Sawyer, R. K. (Ed.). Cambridge handbook of the learning sciences. Cambridge: Cambridge University Press, p. 409-426. Disponível em: <http://gerrystahl.net/cscl/cscl_portugese.htm>. Acesso em: 24 ago. 2015.

TAROUCO, L. M. R.; FABRE, M.C. J. M.; TAMUSIUNAS, F. R. Reusabilidade de objetos educacionais. RENOTE: Revista Novas Tecnologias da Educação, Porto Alegre, v. 1 n. 1, p. 1-11. 2003. Disponível em:<http://www.cinted.ufrgs.br/renote/>. Acesso em: 03 mar. 2015.

WILEY, D. A. (2000) Connecting learning objects to instructional design theory: A definition, a metaphor, and a taxionomy. Disponível em: 〈http://reusability.org/read/chpters/wiley.doc〉. Acesso em: 05 out. 2014. 